\title{
Testing circumstellar disk lifetimes in young embedded clusters associated with the Vela Molecular Ridge ${ }^{\star}$
}

\author{
F. Massi ${ }^{1}$, E. Di Carlo ${ }^{2}$, C. Codella ${ }^{1}$, L. Testi ${ }^{1,3}$, L. Vanzi ${ }^{4}$, and J. I. Gomes ${ }^{5,6}$ \\ 1 INAF - Osservatorio Astrofisico di Arcetri, Largo E. Fermi 5, 50125 Firenze, Italy \\ e-mail: [fmassi; codella]@arcetri.astro.it \\ 2 INAF - Osservatorio Astronomico di Collurania-Teramo, via M. Maggini, 64100 Teramo, Italy \\ e-mail: dicarlo@oa-teramo.inaf.it \\ 3 European Southern Observatory, Karl Schwarzschild str. 2, 85748 Garching, Germany \\ e-mail: 1testi@eso.org \\ 4 Departamento de Ingeniera Electrica, Pontificia Universidad Catolica de Chile, Av. Vicuña Mackenna 4860, Santiago, Chile \\ e-mail: lvanzi@ing.puc.cl \\ 5 Centro de Astronomia e Astrofisica da Universidade de Lisboa, Tapada da Ajuda, 1349-018 Lisboa, Portugal \\ 6 Centre for Astrophysics Research, University of Hertfordshire, College Lane, Hatfield, Hertfordshire, AL10 9AB, UK \\ e-mail: j.gomes@herts.ac.uk
}

Received 14 November 2009 / Accepted 23 February 2010

\begin{abstract}
Context. The Vela Molecular Ridge hosts a number of young embedded star clusters at the same evolutionary stage.

Aims. We test whether the fraction of members with a circumstellar disk in a sample of clusters in the cloud D of the Vela Molecular Ridge, is consistent with relations derived for larger samples of star clusters with an age spread. In addition, we constrain the age of the young embedded star clusters associated with cloud D.

Methods. We carried out $L(3.78 \mu \mathrm{m})$ photometry using images of six young embedded star clusters associated with cloud D of the Vela Molecular Ridge, taken with ISAAC at the VLT. These data are complemented with the available $H K_{\mathrm{s}}$ photometry. The 6 clusters are of roughly the same size and appear to be at the same evolutionary stage. The fraction of stars with a circumstellar disk was measured in each cluster by counting the fraction of sources found to have a NIR excess in colour-colour $\left(H K_{\mathrm{s}} L\right)$ diagrams. Results. The $L$ photometry allowed us to identify the NIR counterparts of the IRAS sources associated with the clusters. The fraction of stars with a circumstellar disk appears to be constant within the errors for the 6 clusters. There is a hint that this is lower for the most massive stars. The age of the clusters is constrained to be $\sim 1-2 \mathrm{Myr}$.

Conclusions. The fraction of stars with a circumstellar disk in the observed sample is consistent with the relations derived from larger samples of star clusters and with other age estimates for cloud D. The fraction may be lower for the most massive stars. Our results agree with a scenario where all intermediate and low-mass stars form with a disk, whose lifetime is shorter for higher mass stars.
\end{abstract}

Key words. stars: formation - stars: pre-main sequence - stars: circumstellar matter -

ISM: individual objects: Vela Molecular Ridge - open clusters and associations: general - infrared: ISM

\section{Introduction}

Open clusters have long been considered as suitable laboratories for testing stellar evolution theories and sampling the initial mass function (IMF). Near-infrared (NIR) cameras have unveiled the precursors of open clusters to be young embedded star clusters. It is even more interesting that a significant fraction of star formation in giant molecular clouds occurs in young embedded clusters. However fewer than $\sim 10 \%$ of these embedded clusters emerge from molecular clouds as bound open clusters (see review by Lada \& Lada 2003). Young embedded clusters are now regarded as excellent sites to measure an IMF, although in these studies one has to take into account the bias introduced by e.g., heavy reddening and the NIR excess exhibited by young pre-main sequence (pms) stars (see, e.g., review by Scalo 1998).

Giant molecular clouds hosting a number of young embedded clusters of the same age represent more valuable laboratories, allowing one to study an even wider range of physical

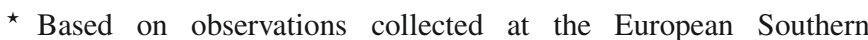
Observatory, Cerro Paranal, Chile, programme 074.C-0630. mechanisms than in individual star clusters. In this respect, one of the most interesting regions is the vela molecular ridge (VMR), first studied in the CO(1-0) by Murphy \& May (1991). The VMR is a giant molecular cloud complex located in the

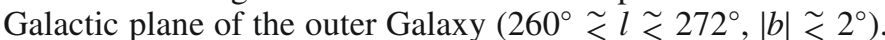
Murphy \& May (1991) subdivided the region into 4 main clouds called A, B, C, and D. Its distance was discussed by Liseau et al. (1992), who concluded that A, C, and D all lie at $700 \pm 200 \mathrm{pc}$. The molecular gas distribution of the VMR was then studied at higher resolution by Yamaguchi et al. (1999). Elia et al. (2007) and Massi et al. (2007) mapped a $1^{\circ} \times 1^{\circ}$ sky area towards cloud $\mathrm{D}$ in the $\mathrm{CO}(1-0)$ and ${ }^{13} \mathrm{CO}(2-1)$ transitions and in the continuum $1.2 \mathrm{~mm}$ emission, respectively, with the SEST. The dynamical structure of the molecular gas suggests an age of $\sim 1 \mathrm{Myr}$ for this part of cloud D. Star formation in clouds A, C, and D (both in young embedded clusters and in isolation) was studied in a number of works (to quote but a few: Liseau et al. 1992; Lorenzetti et al. 1993, 2002; Wouterloot \& Brand 1999; Massi et al. 1999, 2000, 2003; Giannini et al. 2005, 2007; Burkert et al. 2000; Caratti o Garatti et al. 2004; Baba et al. 2004, 2006; 
Table 1. Description of the observed fields.

\begin{tabular}{|c|c|c|c|c|c|}
\hline $\begin{array}{r}\text { IRAS }^{a} \\
\text { source } \\
\end{array}$ & RA(2000) & $\operatorname{Dec}(2000)$ & $\begin{array}{l}\text { Total gas mass }{ }^{b} \\
\left(M_{\odot}\right)\end{array}$ & $\begin{array}{l}\text { Total mass in dense } \text { gas }^{c} \\
\qquad\left(M_{\odot}\right)\end{array}$ & $\begin{array}{c}\text { Cluster stellar mass }{ }^{d} \\
\left(M_{\odot}\right)\end{array}$ \\
\hline IRAS08438-4340 (IRS16) & $08: 45: 35.8$ & $-43: 51: 00$ & 99 & 105 & 103 \\
\hline IRAS08448-4343 (IRS17) & $08: 46: 35.0$ & $-43: 54: 30$ & 48 & 88 & 90 \\
\hline IRAS08470-4243 (IRS18) & 08:48:47.7 & $-42: 54: 22$ & $\left({ }^{e}\right)$ & $\left({ }^{f}\right)$ & 89 \\
\hline IRAS08476-4306 (IRS20) & $08: 49: 26.6$ & $-43: 17: 13$ & 13 & 21 & 70 \\
\hline IRAS08477-4359 (IRS21) & $08: 49: 32.9$ & $-44: 10: 47$ & 219 & 60 & 68 \\
\hline IRAS08485-4419 (IRS22) & 08:50:20.7 & $-44: 30: 41$ & $\left({ }^{e}\right)$ & $\left({ }^{f}\right)$ & $>38^{g}$ \\
\hline
\end{tabular}

Notes. ${ }^{(a)}$ Also listed the nomenclature used by Liseau et al. (1992); ${ }^{(b)}$ derived from $\mathrm{CO}(1-0)$ and ${ }^{13} \mathrm{CO}(2-1)$ observations by Elia et al. (2007); ${ }^{(c)}$ derived from continuum $1.2 \mathrm{~mm}$ observations by Massi et al. (2007); ${ }^{(d)}$ estimated by Massi et al. (2006); ${ }^{(e)}$ found CO(1-0) emission (Liseau et al. 1992); ${ }^{(f)}$ found a dense core at $1.2 \mathrm{~mm}$ centred at the cluster (Massi, private comm.); ${ }^{(g)}$ inferred from the data in Massi et al. (2003).

Apai et al. 2005; Thi et al. 2006; De Luca et al. 2007; Ortiz et al. 2007; Netterfield et al. 2009; Olmi et al. 2009).

Massi et al. (2006) exploited the natural "laboratory" provided by cloud D by selecting a sample of 6 small young embedded clusters in a similar evolutionary stage that they observed in the NIR $J H K_{\mathrm{s}}$ bands. They studied the IMFs and also attempted to constrain the age. The IMFs are both consistent with each other and with a standard IMF (e.g., the one proposed by Scalo 1998). But the cluster age can only be loosely constrained to lie in the interval $\sim 1-5$ Myr. It is therefore important to obtain a more accurate age determination.

A valuable tool in determining the age of young embedded clusters is their content of pms stars with a circumstellar disk, which are identifiable by means of their NIR excess with respect to photospheric emission (Haisch et al. 2001; Hillenbrand 2005). In principle, this content can be easily measured by counting cluster members that exhibit a NIR excess and those in the reddening band of the main sequence, in a $J H K L$ or $H K L$ colourcolour diagram. In the case of the VMR, it is tempting to exploit the large number of young embedded clusters of the same age to also test whether the fraction of stars with a circumstellar disk depends only on the cluster age or whether other effects have to be taken into account. In a scenario where all protostars accrete through a disk, the fraction of stars with a circumstellar disk in a cluster must depend only on disk lifetimes, hence it is expected to be only a function of cluster age, at least in clusters of similar sizes. With these two aims in mind, we selected a sample of 6 young embedded clusters associated with the part of cloud D mapped by Elia et al. (2007) and Massi et al. (2007), and imaged them in the $L(3.78 \mu \mathrm{m})$ band. The photometry obtained was then complemented with already available $H K_{\mathrm{s}}$ photometry.

The paper layout is the following: observations and data reduction are described in Sect. 2, and the results are discussed in Sect. 3 and summarised in Sect. 4.

\section{Observations and data reduction}

We selected the same fields as Massi et al. (2006), except for IRS19 because the NIR counterpart of this IRAS source is too bright in $L$ and would saturate the detector. We replaced it with IRS22, which is another site hosting a young embedded star cluster that is associated with cloud D. These are the most populated clusters in cloud D of the VMR. The six fields are listed in Table 1, along with the gas and stellar masses associated with the stellar clusters. The total gas mass is inferred from Table 3 of Elia et al. (2007), whereas the total mass in dense gas is taken from Table 1 of Massi et al. (2007). We note that the total gas masses listed are less than the total masses in dense gas in three cases. The gas mass is derived in Elia et al. (2007) by using $\mathrm{CO}(1-0)$ and ${ }^{13} \mathrm{CO}(2-1)$ observations (and assuming LTE conditions) of the highest spatial resolution obtained so far. Nevertheless, their ${ }^{13} \mathrm{CO}(2-1)$ observations are spatially undersampled by a factor 2 and their sensitivity is low $(\sim 0.7 \mathrm{~K})$, meaning that the computed masses have to be considered as mere lower limits. Yamaguchi et al. (1999) find much higher gas masses by using $\mathrm{CO}(1-0)$ and ${ }^{13} \mathrm{CO}(1-0)$ observations with a larger beam.

The fields were observed with the camera ISAAC (Moorwood et al. 1999) at the ESO-VLT telescope (Cerro Paranal, Chile) through the broad-band filter $L$ centred on $3.78 \mu \mathrm{m}$, in the LW imaging mode. The pixel scale is $\sim 0.07 \mathrm{arcsec} / \mathrm{pixel}$ and the field of view is $73 \times 73 \mathrm{arcsec}^{2}$. All observations were made in service mode. IRS 17 and IRS 18 were observed on 2005 January 24, IRS 20, IRS 21 and IRS 22 were observed on 2005 January 25 and IRS 16 on 2005 February 20. On each night, images of the standard star HD 75223 (van der Bliek et al. 1996) and dark frames were also taken. Sky flats were acquired on 2005 January 24.

For every field, two sets of 12 chopping sequences of images were taken. In each chopping sequence, the pointing was switched between two different positions (hereby called on and off, although both point towards the target) 30 times, by moving the telescope secondary mirror. The integration time in each on and off position results from an average of (NDIT $=$ ) 9 single exposures of (DIT=) $0.11 \mathrm{~s}$. After each chopping sequence, all ons were averaged together and the same for all offs, then the telescope was nodded, following an ABBAABBA ... sequence. The chop throw was $15^{\prime \prime}$ and the only difference between the two sets of 12 chopping sequences was the throw angle. The first sequence was chopped (in equatorial coordinates) east-west, whereas the second one was chopped 45 degrees to the east-west direction. A small jitter $\left(\sim 5^{\prime \prime}\right)$ was used between ABBA chopping sequences. The nodding was in the same direction as the chopping, with the same throw. The total integration time per field was $\sim 24 \mathrm{~min}$.

Data were reduced using IRAF ${ }^{1}$ routines following the steps outlined in the "ISAAC Data Reduction Guide 1.5", available online. Each on and off frame was first dark subtracted and then corrected for non-linearity according to Sect. 7.4.2 of the Data Reduction Guide (using updated coefficients). After flatfielding, for each single chopping sequence the averaged off frame was subtracted from the averaged on frame. All obtained on-off frames were then corrected for bad pixels.

\footnotetext{
1 IRAF is distributed by the National Optical Astronomy Observatories, which are operated by the Association of Universities for Research in Astronomy, Inc., under cooperative agreement with the National Science Foundation.
} 
An on-off frame consists of displaced "positive" and "negative" images of stars (due to the chop throw). Each AB cycle yields four different shifted images of the same field, i.e. two onoff and two off-on. For each AB cycle, by subtracting the on-off $\mathrm{B}$ from the on-off $\mathrm{A}$ one obtains a new image in which residual sky patterns are effectively cancelled. However, there are now two symmetric "negative" stars with respect to the corresponding positive ones (twice in counts). This image can be multiplied by -1 and shifted to obtain two more independent images (also with negative stars). Therefore, we produced 4 sky-subtracted, shifted images for each AB cycle. Finally, all sky-subtracted images of a same field were registered and averaged together, after removal of the "negative" stars, to yield the final image. We also constructed a median final image in the same way, and an average and a median image for each of the two sets with different chopping throw angle. We note that, because of chopping and nodding, only the very central area of the final image exploits the full $\sim 24 \mathrm{~min}$ of integration. The outermost image area was exposed for 6 minutes. The effective integration time increases from the edge to the centre.

The seeing was quite good: we found average PSF FWHMs of $\sim 0.3^{\prime \prime}$ (IRS16), $\sim 0.5^{\prime \prime}$ (IRS17 and IRS18), and $\sim 0.4-0.6^{\prime \prime}$ (IRS20, IRS21 and IRS22). Photometry on the $L$ images was performed by using DAOPHOT tasks in IRAF. For each field, the list of detections obtained with DAOFIND was visually checked in the final median images, to search for both false and missed detections. It was very useful having images with different chopping throw angles to check for stars that might have been cancelled in the preliminary on-off subtraction. Aperture photometry was carried out with PHOT, by using an aperture radius $\sim 1 F W H M$ and inner and outer radii for the sky annulus of $\sim 2$ and $\sim 4 F W H M$ s. Finally, we performed PSF-fitting photometry with ALLSTAR.

For IRS16, IRS17, IRS18, IRS20, and IRS21, we complemented our $L$ photometry with the $J H K_{\mathrm{s}}$ photometry (from SofI images taken at NTT/ESO) of Massi et al. (2006). We crosschecked both photometries and redid the $J H K_{\mathrm{s}}$ photometry for a few sources that had gone undetected by Massi et al. (2006). In the case of IRS17, the group of sources towards \# 40 of Massi et al. (1999), resolved on the ISAAC image, are barely resolved on the SofI images. Hence, only for the group of stars close to \# 40 did we adopt the $J H K_{\text {s }}$ photometry (from ISAAC images taken at the VLT) by Giannini et al. (2005).

For IRS22, we performed new $H K_{\mathrm{s}}$ photometry on SofI images taken on 2007 December 31 as part of ESO programme 080.C-0836. These used the Large Field imaging mode (as in Massi et al. 2006) with a plate scale of $\sim 0.29 \mathrm{arcsec} /$ pixel, yielding a f.o.v. of $4.9 \times 4.9 \operatorname{arcmin}^{2}$. At both $H$ and $K_{\mathrm{s}}$, the field was imaged in a $2 \times 2$ square grid, chosen in such a way that all frames overlap on a central area $\sim 2 \times 2$ arcmin $^{2}$ containing the cluster. For each of the 4 positions, 10 dithered integrations were performed, each one a mean of 12 exposures of $2 \mathrm{~s}$. After each on-source integration, the telescope was moved to a location off-source and a sky image taken with the same averaged exposure time. Dome flat field images were taken before the observations. Data reduction was carried out by using standard IRAF routines. All frames were corrected for cross-talk and flat-fielded. For each on-source frame, a sky frame was constructed by median-combining the 4 closest (in time) off-source frames after star removal. All on-source frames were then skysubtracted and corrected for bad pixels. Finally, they were registered and combined by obtaining their average. The $L$-field is contained in the central $\sim 2 \times 2 \operatorname{arcmin}^{2}$ area of the $H K_{\mathrm{s}}$ mosaics, so the total integration time of interest is $16 \mathrm{~min}$.
Photometry in the $H K_{\mathrm{s}}$ band was carried out with DAOPHOT tasks in IRAF. We searched for stars with DAOFIND and, then, visually inspected the images to remove false detections and/or add missed detections (after a cross-check with the $L$ image). We used PHOT to perform aperture photometry, with an aperture radius of $\sim 1 F W H M$ and inner and outer sky annuli of $\sim 2$ and $\sim 4 F W H M$ s (assuming the median to be an estimate of the sky). The PSF FWHM is $\sim 0.9^{\prime \prime}$. Calibration was obtained by using standard stars imaged during the night from the list of Persson et al. (1998).

We finally combined the $J H K_{\mathrm{s}}$ and $L$ photometry. Over the total solid angle imaged by ISAAC towards each cluster, the number of $L$ sources with an identified $K_{\mathrm{S}}$ counterpart is: 107 (IRS16), 67 (IRS17), 133 (IRS18), 49 (IRS20), 71 (IRS21), and 101 (IRS22). The number of $L$ sources without a $K_{\mathrm{s}}$ counterpart is instead: 13 (IRS22), 2 (IRS16), 1 (IRS17), 1 (IRS18), and 0 (IRS20 and IRS21). The number of $K_{\mathrm{S}}$ sources without an $L$ counterpart is: 34 (IRS16), 65 (IRS17), 87 (IRS18), 109 (IRS20), 48 (IRS21), and 105 (IRS22). If $K_{\mathrm{s}}-L$ were computed for the $K_{\mathrm{s}}$ sources without an $L$ counterpart by using the $L$ completeness limits estimated in Sect. 3.1, most of these objects would fall to the right of the main sequence reddening band in a $H-K_{\mathrm{s}}$ versus $K_{\mathrm{s}}-L$ diagram, that is they are consistent with reddened or unreddened stars whose $L$ photospheric emission lies below the completeness limit (in flux). Some of them might even have a NIR excess and still go undetected in $L$. This also means that the available $K_{\mathrm{s}}$ photometry is deeper (in terms of sampled stellar masses) than our $L$ photometry.

\section{Results}

\subsection{Completeness limits}

To estimate the completeness limit of our photometry, we first constructed histograms of the number of sources versus $L$ for each field, binning $L$ in 0.5 mag intervals. In all histograms, the number of sources increases with increasing magnitude, reaches a peak, and then decreases. The peak is the signature of the efficiency in finding sources starting to decrease. Using the task ADDSTAR in DAOPHOT, we carried out experiments by adding small sequences of artificial stars randomly distributed over each frame. We could always retrieve more than $80 \%$ of the artificial stars at the central magnitude of each histogram peak. Furthermore, the magnitudes measured with aperture photometry (PHOT) were within $\sim 0.1$ mag of the true values about the peak magnitude.

Hence, we estimated completeness magnitudes of $L_{\mathrm{compl}} \sim$ 14.25 (IRS16, IRS21, and IRS22), $L_{\text {compl }}^{\prime} \sim 13.75$ (IRS 17 and IRS 18), and $L_{\text {compl }}^{\prime \prime} \sim 13.25$ (IRS 20), which are $1-1.5$ mag below the detection limits $(L \sim 14.5-15.7)$. The detection limits were estimated by examining the error versus magnitude diagrams and locating the faintest stars in each diagram. The most populous clusters, IRS18 and IRS22, also exhibit a second peak in the number of sources versus $L$, located $\sim 2$ mag below the completeness magnitude, which we interpret as an intrinsic feature of the cluster population.

Using the assumed distance modulus to Vela-D (9.225 mag) and an estimate of the maximum extinction, we can convert $L_{\text {compl }}\left(L_{\text {compl }}^{\prime}, L_{\text {compl }}^{\prime \prime}\right)$ into a limiting absolute magnitude. This can then be converted into a limiting stellar mass if a maximum age for the cluster members is also assumed. By using the reddening law by Rieke \& Lebofsky (1985), we derived completeness limits in absolute magnitudes of $L \sim 4.45$ $(\sim 3.95,3.45)$ for $A_{\mathrm{V}} \sim 10 \mathrm{mag}$, and $L \sim 2.71(\sim 2.21,1.71)$ for 


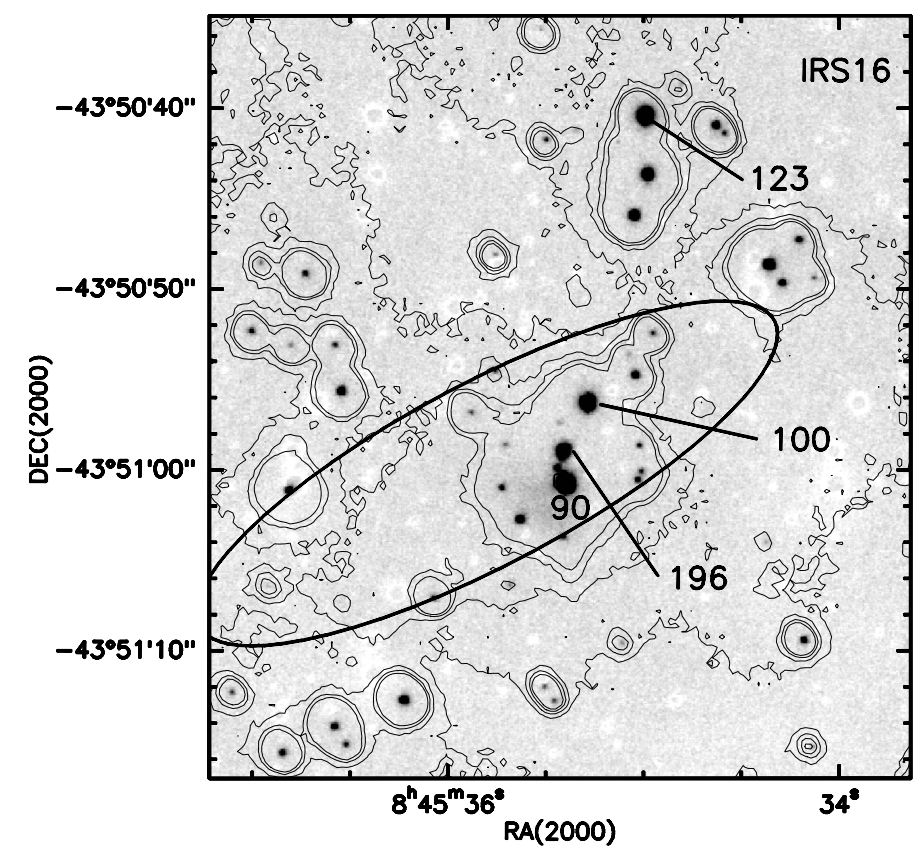

Fig. 1. $L$-band image of the inner $\sim 1 \times 1 \mathrm{arcmin}^{2}$ region of the cluster associated with IRS16. This is overlaid with contours of the emission in the $K_{\mathrm{s}}$-band (from the SofI image by Massi et al. 2006). Contours range from $\sim 10 \sigma$ of the background in steps of $\sim 10 \sigma$. The IRAS uncertainty ellipse is also drawn, and the most prominent NIR sources identified by Massi et al. (2003) are labelled according to the numbers these authors assigned in their list.

$A_{\mathrm{V}} \sim 40$ mag. To convert these magnitudes into stellar masses, we used pms evolutionary tracks by a number of different authors. Adopting the tracks by Palla \& Stahler (1999), by a rough comparison with the colours given by Koornneef (1983) and Bessell \& Brett (1988), the values for $A_{\mathrm{V}} \sim 40$ mag are consistent with $M \sim 0.4 M_{\odot}\left(\sim 0.6,1 M_{\odot}\right)$ for an age of $1 \mathrm{Myr}$, and $M<0.1 M_{\odot}\left(<0.2, \sim 0.2 M_{\odot}\right)$ for an age of 0.1 Myr. Adopting the tracks by D'Antona \& Mazzitelli (1994), these numbers become $M \sim 0.6 M_{\odot}\left(\sim 0.8,1 M_{\odot}\right)$ for an age of $1 \mathrm{Myr}$, and $M \sim 0.2 M_{\odot}\left(\sim 0.3,0.4 M_{\odot}\right)$ for an age of $0.1 \mathrm{Myr}$.

The tracks by Siess et al. (2000), in addition to the colours of Kenyon \& Hartmann (1995), yield $M \sim 0.2 M_{\odot}\left(\sim 0.3,0.7 M_{\odot}\right)$ for an age of $1 \mathrm{Myr}$ and $M<0.1 M_{\odot}\left(\sim 0.2,0.3 M_{\odot}\right)$ for an age of 0.1 Myr. Finally, the tracks of Baraffe et al. (1998) yield $M \sim 0.13 M_{\odot}$ for an age of $2 \mathrm{Myr}$, if the colours by Kenyon \& Hartmann (1995) are used. However, these colours appear to be inconsistent with those inferred from Baraffe et al. (1998). Using the colours given by Koornneef (1983), one obtains $M$ $0.7 M_{\odot}$. In summary, the $L$ completeness limits correspond to stellar masses in the range $0.1-0.7 M_{\odot}\left(0.2-0.8,0.2-1 M_{\odot}\right)$, seen through an extinction of $A_{\mathrm{V}} \sim 40 \mathrm{mag}$. We note that this takes into account the star photometric emission only, so lower mass stars remain detectable if they exhibit a NIR excess.

\subsection{The observed fields}

Zoomed images of the inner $\sim 1 \times 1 \operatorname{arcmin}^{2}$ fields in the $L$-band are shown in Figs. 1 to 6 . The $L$ images are overlaid with contours of the lowest levels of the emission in $K_{\mathrm{s}}$ (i.e., starting from $\sim 10 \sigma$ of the background) measured on SofI (IRS16, IRS17, IRS18, IRS20, IRS21; Massi et al. 2006; IRS22, this work) images to enhance the differences between the two bands. The diffuse emission in the $L$ band is clearly not as prominent as in the

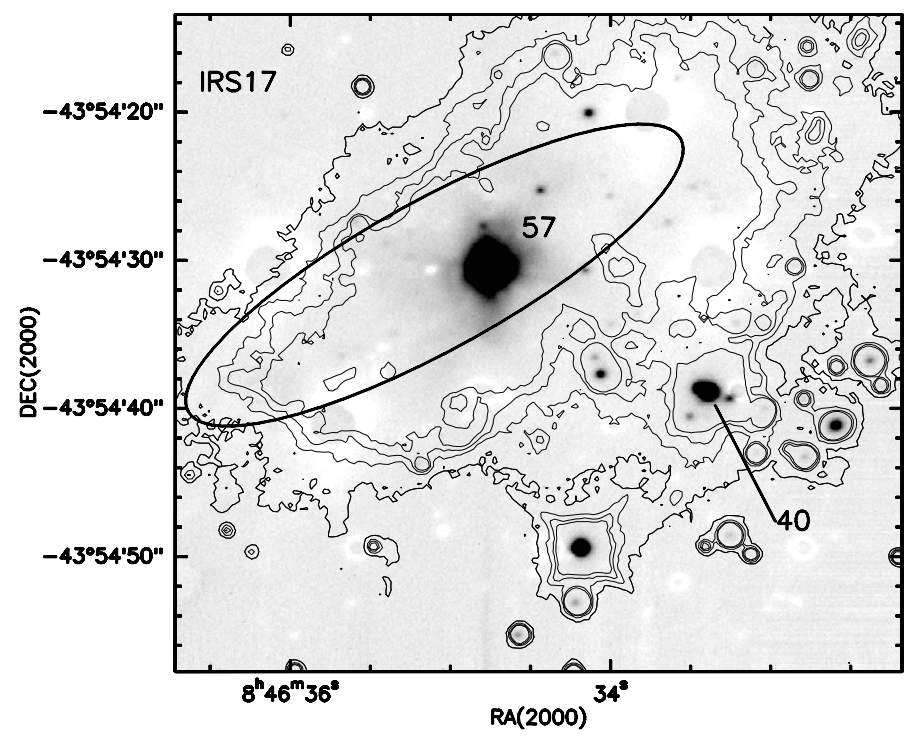

Fig. 2. $L$-band image of the inner $\sim 1 \times 1 \operatorname{arcmin}^{2}$ region of the cluster associated with IRS17. This is overlaid with contours of the emission in the $K_{\mathrm{s}}$-band (from the SofI image by Massi et al. 2006). Contours range from $\sim 10 \sigma$ of the background in steps of $\sim 10 \sigma$. The IRAS uncertainty ellipse is also drawn, and the most prominent NIR sources identified by Massi et al. (1999) are labelled according to the numbers these authors assigned in their list.

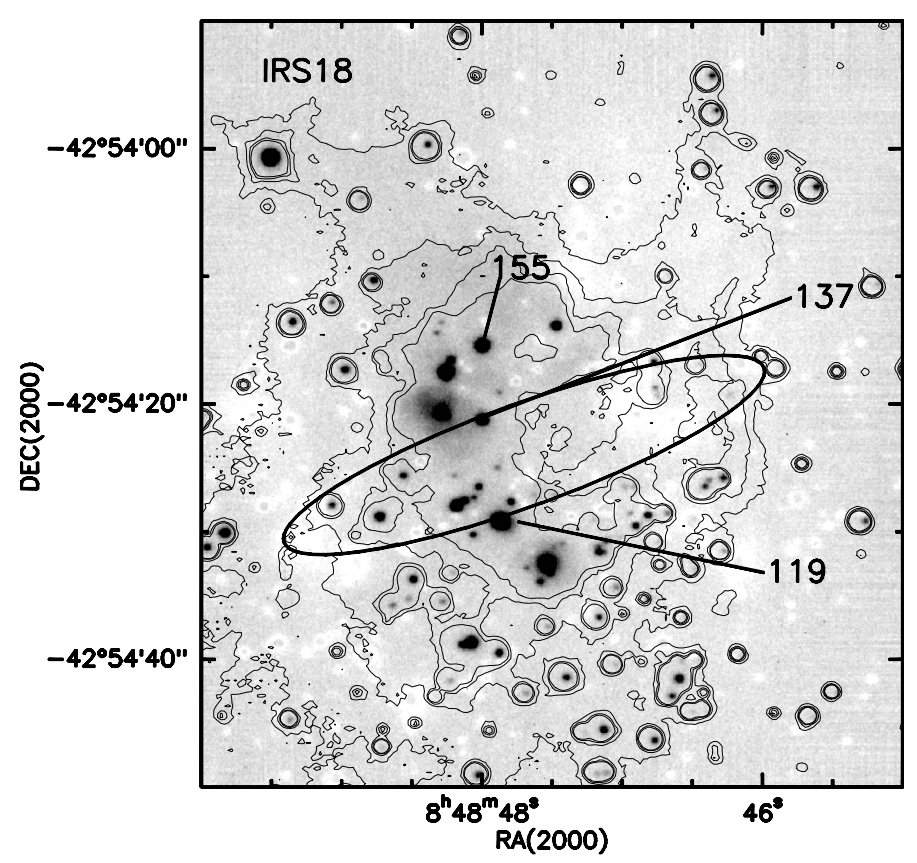

Fig. 3. $L$-band image of the inner $\sim 1 \times 1 \operatorname{arcmin}^{2}$ region of the cluster associated with IRS18. This is overlaid with contours of the emission in the $K_{\mathrm{s}}$-band (from the SofI image by Massi et al. 2006). Contours range from $\sim 10 \sigma$ of the background in steps of $\sim 10 \sigma$. The IRAS uncertainty ellipse is also drawn, and the most prominent NIR sources identified by Massi et al. (1999) are labelled according to the numbers these authors assigned in their list.

$K_{\mathrm{s}}$ band. The spatial resolution in $L$ also appears to be higher than in $K_{\mathrm{s}}$. Furthermore, the brightest $K_{\mathrm{s}}$ sources do not appear to be saturated in $L$. All of this enables us to resolve sources in $L$ that are not resolved in $K_{\mathrm{s}}$ or lie within intense diffuse emission. We also note that we have higher spatial resolution, and none of the sources is saturated, with respect to Spitzer data. 
F. Massi et al.: Testing circumstellar disk lifetimes in young embedded clusters associated with the Vela Molecular Ridge

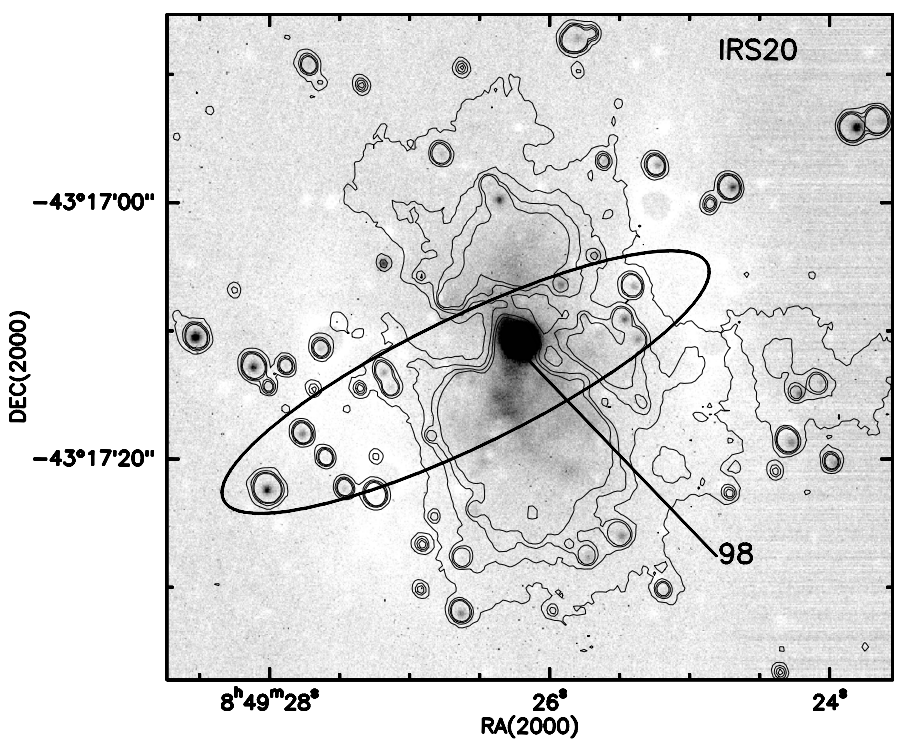

Fig. 4. $L$-band image of the inner $\sim 1 \times 1 \operatorname{arcmin}^{2}$ region of the cluster associated with IRS20. This is overlaid with contours of the emission in the $K_{\mathrm{s}}$-band (from the SofI image by Massi et al. 2006). Contours range from $\sim 10 \sigma$ of the background in steps of $\sim 10 \sigma$. The IRAS uncertainty ellipse is also drawn, and the most prominent NIR sources identified by Massi et al. (1999) are labelled according to the numbers these authors assigned in their list.

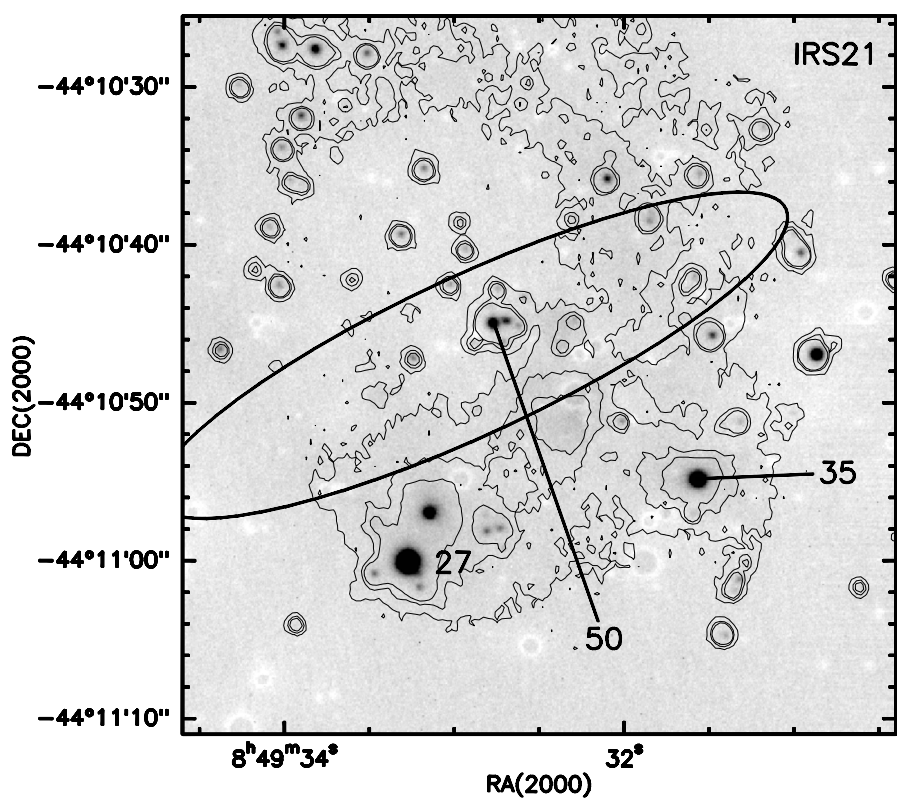

Fig. 5. $L$-band image of the inner $\sim 1 \times 1 \operatorname{arcmin}^{2}$ region of the cluster associated with IRS21. This is overlaid with contours of the emission in the $K_{\mathrm{s}}$-band (from the SofI image by Massi et al. 2006). Contours range from $\sim 10 \sigma$ of the background in steps of $\sim 10 \sigma$. The IRAS uncertainty ellipse is also drawn, and the most prominent NIR sources identified by Massi et al. (1999) are labelled according to the numbers these authors assigned in their list.

A clear example is the central bright source found by Massi et al. (2003) in IRS16 and barely resolved into two stars, their \# 90 and \# 196. Our $L$ image separates the two objects and further shows a fainter star in-between them. The two $L$ stars without a $K_{\mathrm{s}}$ counterpart also both lie towards this group of stars. In the $L$ image of IRS22, source \# 111 of Massi et al. (2003) appears to be composed of two close-by stars. Furthermore, four of the $L$

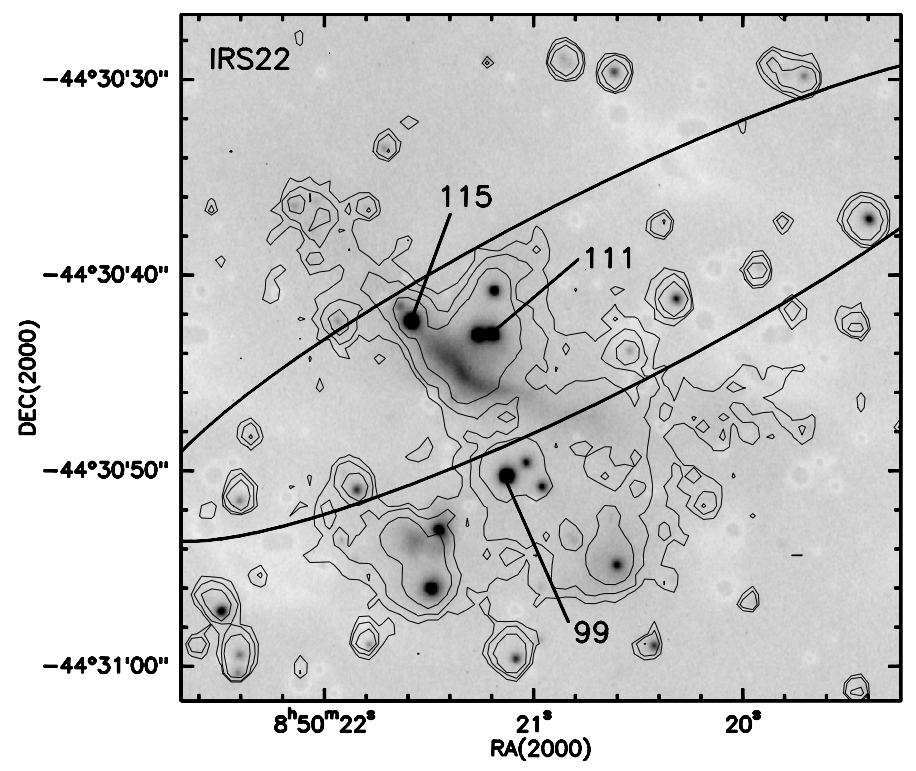

Fig. 6. $L$-band image of the inner $\sim 1 \times 1 \operatorname{arcmin}^{2}$ region of the cluster associated with IRS22. This is overlaid with contours of the emission in the $K_{\mathrm{s}}$-band (from the SofI image taken in 2008). Contours range from $\sim 10 \sigma$ of the background in steps of $\sim 5 \sigma$. The IRAS uncertainty ellipse is also drawn, and the most prominent NIR sources identified by Massi et al. (2003) are labelled according to the numbers these authors assigned in their list.

stars without a $K_{\mathrm{s}}$ counterpart lie towards the compact group of sources around \# 111.

\subsection{The cluster stellar population}

To study the stellar population of the clusters, we computed the NIR colours of all sources with $L<14.25$ (the estimated completeness limit in $L$ for half of the clusters, see Sect. 3.1) and plotted them in a $H-K_{\mathrm{s}}$ versus $K_{\mathrm{s}}-L$ diagram. These diagrams are drawn in Fig. 7 for all fields. To discriminate between sources with a NIR excess and reddened stars, a knowledge of both the colours of unreddened main sequence stars and the reddening law is needed. According to the ISAAC manual, the available $L$ filter is more similar to the $L^{\prime}$ filter described in Bessell \& Brett (1988). According to their Table II, $K-L$ and $K-L^{\prime}$ can differ by up to $\sim 0.1 \mathrm{mag}$ for main sequence $\mathrm{M}$ stars. In contrast, the values of $H-K$ are mostly within $\sim 0.01$ mag of those given by Koornneef (1983). Hence, we adopted the colours (and $L^{\prime}$ ) by Bessell \& Brett (1988) for the main sequence locus drawn in Fig. 7. However, note that the 6 fields were imaged in the $K_{\mathrm{s}}$ (SofI) band, which differs slightly from the $K$ band adopted by Bessell \& Brett (1988) or Koornneef (1983). According to Persson et al. (1998), the scatter in the relationship between $K_{\mathrm{s}}-K$ and stellar colours is caused mostly by a stellar CO-band absorption that affects the $K$ filter more than the $K_{\mathrm{s}}$ filter. For the standards listed in their Table 2, the differences between $K$ and $K_{\mathrm{s}}$ are at most less than $\sim 0.01 \mathrm{mag}$. Differences between $K$ and $K_{\mathrm{S}}$ can be also derived by using the transformations given by Carpenter (2001) for 2 MASS. In the colour range spread by main sequence stars, the differences between $K_{\mathrm{s}}$ (2MASS) and $K$ (in the Koornneef or Bessell systems) are always $<0.05$ mag. These should be of the same order for the SofI filter, so the main sequence colours given by Bessell \& Brett (1988) should be within $\sim 0.05 \mathrm{mag}$ at most of the true values on the SofI 


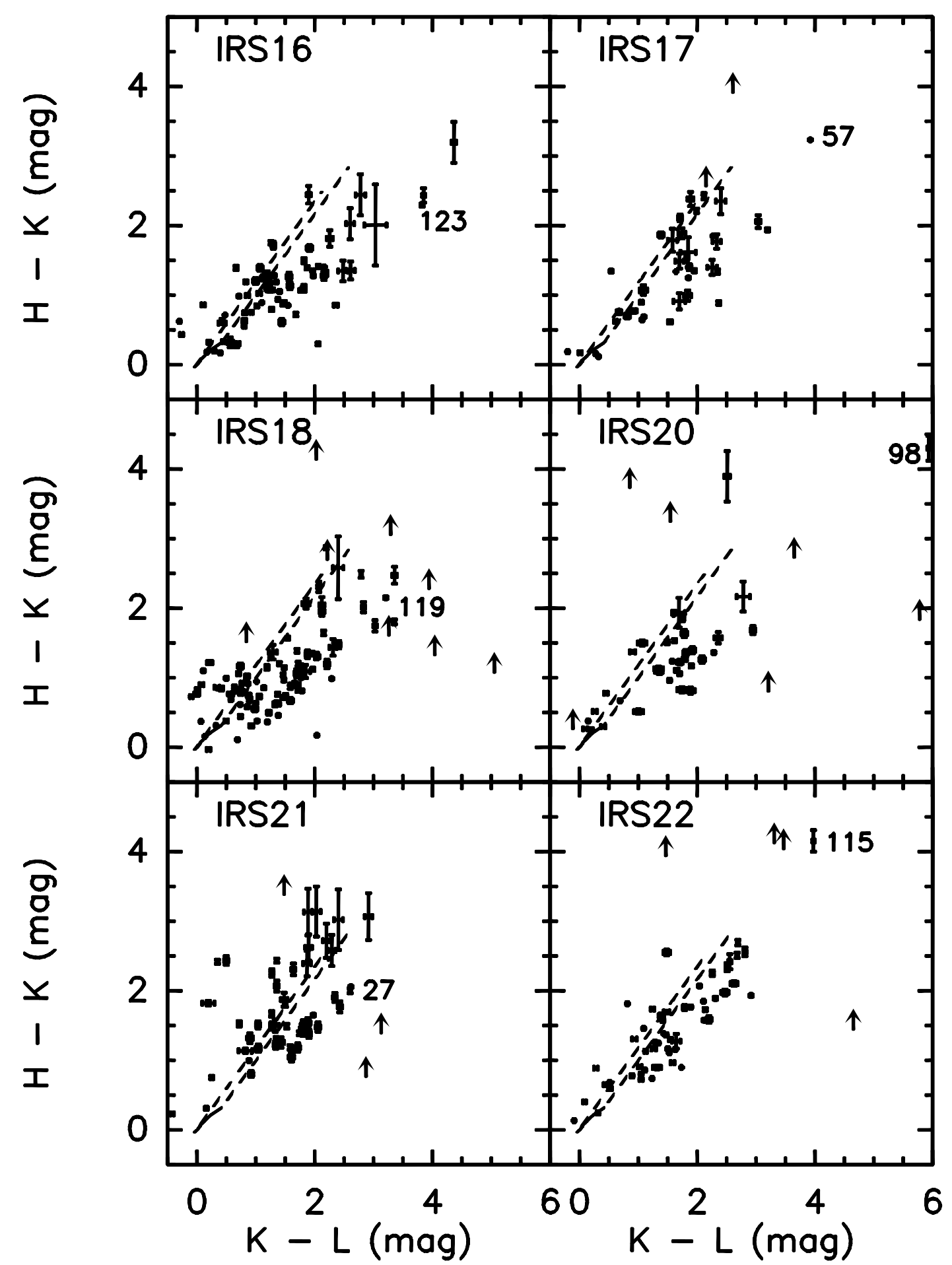

Fig. 7. $H-K_{\mathrm{s}}$ versus $K_{\mathrm{s}}-L$ diagrams for all sources with $L<14.25$ (the completeness limit for half of the clusters) in each field. Arrows mark lower limits in $H-K_{\mathrm{s}}$. The locus of main sequence stars (Bessell \& Brett 1988) is drawn (full line) along with the loci of reddened B8 and M 5 dwarfs up to $A_{\mathrm{V}}=40 \mathrm{mag}$ (dashed lines) according to the law of Rieke \& Lebofsky (1985). A few remarkable sources discussed in the text are labelled using the numbers in the catalogues of Massi et al. $(1999,2003)$.

system. The adopted reddening law is that of Rieke \& Lebofsky (1985), which Massi et al. (2006) showed to be consistent with the reddening in the SofI $J H K_{\mathrm{s}}$ system.

In all the colour-colour diagrams in Fig. 7, it is quite evident that part of the sources are aligned within the reddening band of the main sequence, whereas a large fraction of sources exhibit a NIR excess although they are also spread along a direction parallel to the reddening vector. The NIR counterparts to the IRAS sources (reidentified in Appendix A) are usually located at the upper edge of this sequence of sources with a NIR excess, or of its projection towards the upper-right corner of the diagram (e.g., source \# 57 in IRS17 and source \# 98 in IRS20), meaning they are also heavily reddened. In this respect, source \# 98 (IRS20) is by far the most extincted of all counterparts $\left(A_{\mathrm{V}} \sim 80 \mathrm{mag}\right)$, consistent with it being viewed through an edge-on circumstellar disk. 

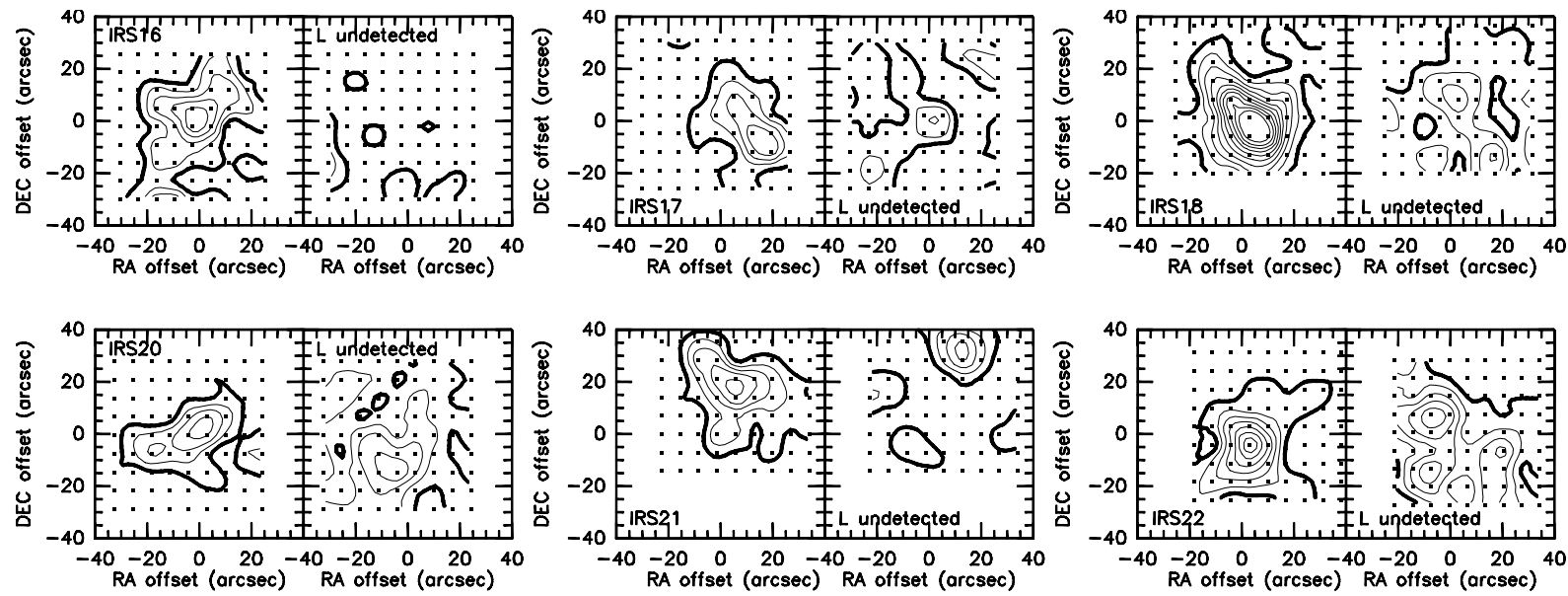

Fig. 8. Maps of the surface density of stars towards the observed fields. In the left-hand boxes, the surface density of sources with $L<14.25$ is plotted, whereas in the right-hand boxes the surface density of $K_{\mathrm{s}}$ sources without an $L$ counterpart is shown. The lowest contour (thick line) is $40 \mathrm{stars} \operatorname{arcmin}^{-2}$, the other contours are in steps of $40 \mathrm{stars} \operatorname{arcmin}^{-2}$. Coordinates are offsets from the position of the identified counterparts of the IRAS sources.

Datapoints are also found above the reddening band of the main sequence in all fields. We found that most of these sources are very close to other sources in the $K_{\mathrm{s}}$ frames, so that the aperture photometry in $K_{\mathrm{s}}$ is contaminated by the light of the nearby stars and the corresponding $K_{\mathrm{s}}-L$ values are also moved leftwards. The sources within the reddening band of the main sequence exhibit extinctions up to $A_{\mathrm{V}}=20-40 \mathrm{mag}$. This and the high fraction of sources with a NIR excess, indicate that the fields imaged in $L$ are dominated by the young members of the embedded clusters.

To prove that the imaged $L$ fields are the cluster cores, we plot the surface density of $L$ sources in Fig. 8 for all the fields (left boxes). The surface density is obtained by counting all sources with $L<14.25$ within $14^{\prime \prime}$-side squares, displaced by $7^{\prime \prime}$ in right ascension and declination from each other. The surface density maps are centred on the identified NIR counterparts of the IRAS sources (see Appendix A). By binning together all counts per cell towards the 6 clusters, we obtained a histogram that is reminiscent of a Poissonian curve with 1 average and an excess of counts on the wing. This allows us to roughly estimate a standard deviation of 20 stars $\operatorname{arcmin}^{-2}$ towards all fields. All cluster cores clearly fall within the imaged fields. We note that some of the proposed counterparts are not located at the centre of the clusters (e.g., source \# 57 in IRS17).

\subsection{The population of NIR stars without an L counterpart}

In Sect. 2, we noted the presence of $K_{\mathrm{s}}$ sources without an $L$ counterpart in all fields and checked that they are consistent with faint stars whose $L$ flux falls below the completeness limit. This is confirmed by the magnitude-colour diagrams ( $K_{\mathrm{s}}$ versus $H-K_{\mathrm{s}}$ ) shown in Fig. 9 for all $K_{\mathrm{s}}$ sources in the imaged fields without an $L$ counterparts. All these sources are actually fainter than $K_{\mathrm{s}} \sim 14-15$.

To confirm their nature, in Fig. 8 we plot contours of the surface density of $K_{\mathrm{s}}$ sources without an $L$ counterpart (right boxes) for all fields, obtained as explained above. Some of the fields exhibit a clustering of these sources that is not centred towards the spatial distribution centre of the $L$ sources. However, we note that the brightness of most of these $K_{\mathrm{s}}$ sources is below the completeness limit in $K_{\mathrm{s}}$. Some of these faint sources may in addition be cluster members, and their different spatial distribution may just reflect variations in the detection efficiency due to variations in extinction and diffuse emission across the fields.

\subsection{Contamination of the cluster members by field stars}

To check how much the population of $L$ sources is contaminated by field stars, we derived the $K$ luminosity function (KLF) of each cluster by using all $K_{\mathrm{s}}$ sources with an $L$ counterpart. These KLFs were then dereddened according to the method discussed in Massi et al. (2006), and compared with those obtained for the same clusters by these authors after dereddening and correction for field stars (excluded IRS22, not observed by Massi et al. 2006). Figure 10 shows that all pairs of KLFs are quite similar for all 5 fields at least to $K_{\mathrm{s}}=14$, i.e., the completeness limit estimated by Massi et al. (2006) for their dereddened KLFs. Hence, the $L$ photometry also samples the KLFs to a dereddened $K_{\mathrm{s}}=14$. Some differences are evident at the high luminosity ends, where the statistics are however poor. A $\chi^{2}$ test on each pair of KLFs shows that they are equal at a significance level $>70 \%$. This indicates that the $L$ sources fully sample the cluster population and that the contamination by field stars is very low.

\section{Discussion}

\subsection{Finding the stars with a circumstellar disk}

The selected clusters are a suitable sample to check whether the fraction of members with circumstellar disks depends on only the cluster age or other effects have to be accounted for. First, a number of results suggest that all 6 clusters are roughly equally old:

1. they are all associated with molecular gas (Massi et al. 1999, 2003, 2006) (see Table 1);

2. they are all associated with dense molecular cores (Massi et al. 2007) (see Table 1);

3. they are all associated with a FIR point source that has been identified (except for IRS16) as an intermediate-mass star progenitor (Massi et al. 1999, 2003);

4. they have roughly the same projected size (Massi et al. 1999, 2003);

5. Massi et al. (2006) showed that the best fits to their KLFs are obtained with pms stars of an age $\sim 1-5 \mathrm{Myr}$; 


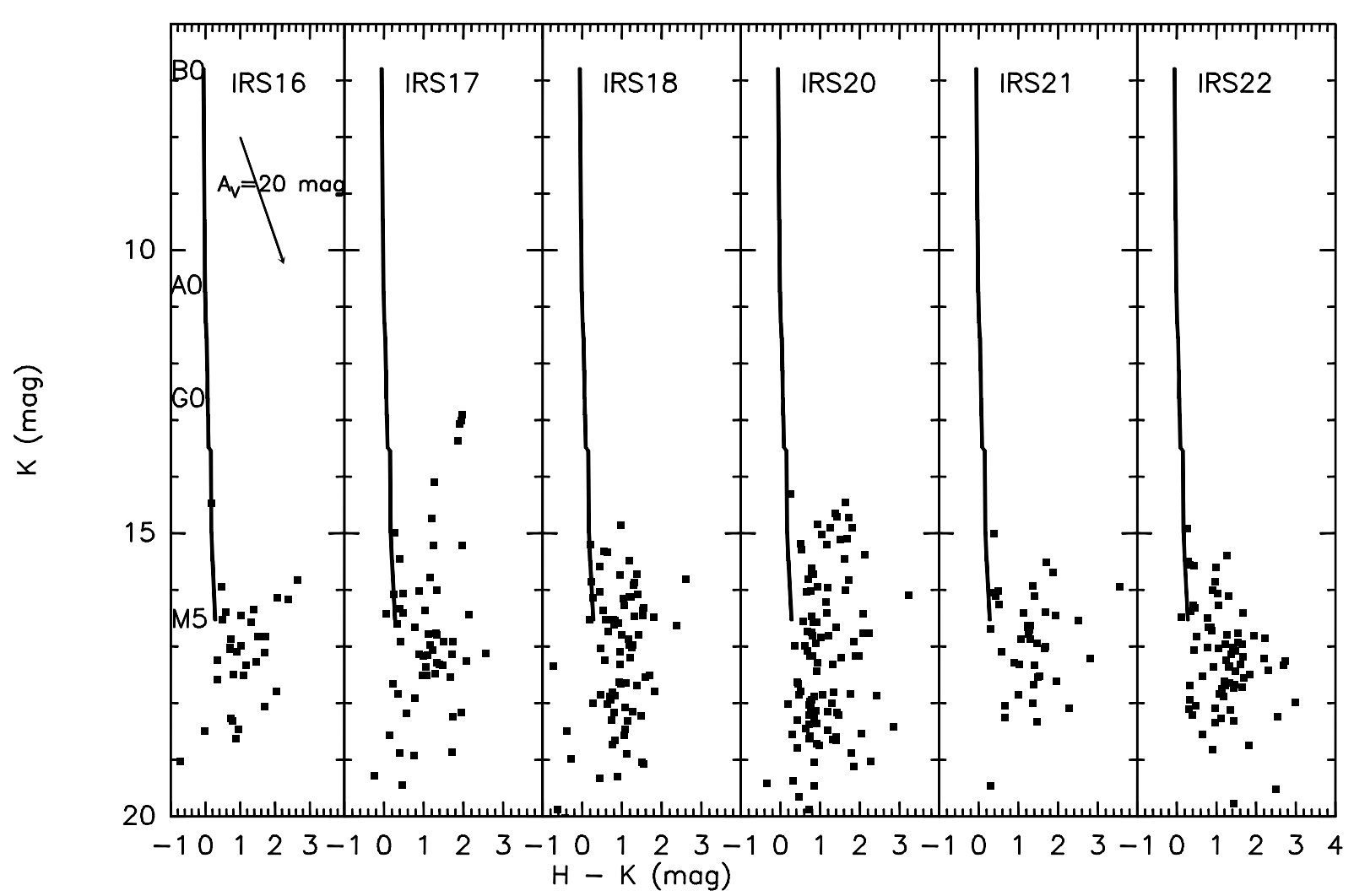

Fig. 9. $K_{\mathrm{s}}$ versus $H-K_{\mathrm{s}}$ diagrams for all $K_{\mathrm{s}}$ sources in the observed fields without an $L$ counterpart. The locus of the ZAMS at $d=700 \mathrm{pc}$ is also drawn, adopting the colours of Koornneef (1983) and the absolute magnitudes given by Allen (1976). A few spectral types are labelled and a reddening vector $A_{\mathrm{V}}=20 \mathrm{mag}$ is drawn, as well, according to Rieke \& Lebofsky (1985).

Table 2. Fraction of $H K_{\mathrm{s}} L$ sources with a NIR excess. We also list the fraction derived by selecting only $H K_{\mathrm{s}} L$ sources whose $K_{\mathrm{s}}$, after correction for reddening as explained in Sect. $4.3\left(K_{\text {dered }}\right)$, is less than given thresholds (the selection criteria for $L$ and $K_{\text {dered }}$ are indicated in the first line).

\begin{tabular}{cccccccc}
\hline \hline & \multicolumn{2}{c}{$L<L_{\text {compl }}^{\prime \prime}$} & \multicolumn{2}{c}{$L<L_{\text {compl }}$} & \multicolumn{2}{c}{$L<L_{\text {compl }}$ and $K_{\text {dered }}<14$} & $L<L_{\text {compl }}$ and $K_{\text {dered }}<11$ \\
Field & Fraction & Fraction & Fraction & Fraction & Fraction & Fraction & Fraction \\
name & corrected & uncorrected & corrected & uncorrected & corrected & uncorrected & uncorrected \\
\hline IRS16 & $0.74 \pm 0.18$ & $0.65 \pm 0.16$ & $0.65 \pm 0.13$ & $0.60 \pm 0.11$ & $0.60 \pm 0.12$ & $0.56 \pm 0.11$ & $0.46 \pm 0.25$ \\
IRS17 & $0.93 \pm 0.30$ & $0.65 \pm 0.23$ & $0.66 \pm 0.18$ & $0.57 \pm 0.15$ & $0.59 \pm 0.16$ & $0.50 \pm 0.14$ & $0.56 \pm 0.31$ \\
IRS18 & $0.73 \pm 0.13$ & $0.68 \pm 0.13$ & $0.66 \pm 0.11$ & $0.63 \pm 0.10$ & $0.62 \pm 0.11$ & $0.59 \pm 0.10$ & $0.43 \pm 0.21$ \\
IRS20 & $0.91 \pm 0.28$ & $0.68 \pm 0.23$ & $0.73 \pm 0.17$ & $0.63 \pm 0.15$ & $0.66 \pm 0.17$ & $0.56 \pm 0.15$ & $0.25 \pm 0.28$ \\
IRS21 & $0.69 \pm 0.22$ & $0.52 \pm 0.19$ & $0.49 \pm 0.12$ & $0.43 \pm 0.11$ & $0.46 \pm 0.11$ & $0.41 \pm 0.11$ & $0.29 \pm 0.16$ \\
IRS22 & $0.87 \pm 0.24$ & $0.66 \pm 0.20$ & $0.69 \pm 0.16$ & $0.62 \pm 0.13$ & $0.67 \pm 0.15$ & $0.60 \pm 0.14$ & $0.50 \pm 0.22$ \\
\hline
\end{tabular}

6. Elia et al. (2007) found that four of the clusters are located within filaments of molecular gas, whose origin is probably dynamical with an estimated age of $\sim 1 \mathrm{Myr}$.

Secondly, all clusters belong to a same region (Vela-D) and are, therefore, at a similar distance and experience a similar reddening distribution. All of them were observed in $J H K_{\mathrm{s}} L$ by using the same instruments and instrumental setups. Hence, they are all affected by the same environmental and instrumental biases and their properties are easier to compare.

Haisch et al. (2000), Lada et al. (2000) and Lada \& Lada (2003) showed that virtually every source with a circumstellar disk exhibits a NIR excess in a $J H K L$ diagram. Hence, the fraction of young stars with a circumstellar disk can be derived by just counting the fraction of objects with a NIR excess in a $J H K L$ diagram. We used the $H K_{\mathrm{s}} L$ diagrams of Fig. 7 , since many $H K_{\mathrm{s}} L$ sources are not detected in $J$. We adopted the main sequence locus of Bessell \& Brett (1988) and the reddening law of Rieke \& Lebofsky (1985), as discussed in Sect. 3.3. Within a given $H-K_{\mathrm{s}}$ versus $K_{\mathrm{s}}-L$ diagram, we conservatively considered as sources with a NIR excess those lying below a line parallel to the reddening vector, passing through a point $0.1 \mathrm{mag}$ bluer in $H-K_{\mathrm{s}}$ than an M5 star. The most optimistic mass limit estimate (with $A_{\mathrm{V}} \sim 40 \mathrm{mag}$ ) corresponding to our completeness $L$ magnitude, discussed in Sect. 3.1, matches a main sequence M5 star, that is, according to Baraffe \& Chabrier (1996) the mass of an M5 V star is $\sim 0.13 M_{\odot}$. In principle, less extincted low mass stars (later than M5) could be detected and counted as stars with a NIR excess. Adopting a selection cut of a lower $L$, e.g., at $L_{\text {compl }}^{\prime \prime}$, significantly reduces this kind of contamination. Furthermore, the obtained fraction of stars exhibiting a NIR excess is likely to be a lower limit. Whereas the $H$ and $K_{\mathrm{S}}$ images were usually taken under similar seeing conditions, the $L$ ones are characterised by higher spatial resolution. Hence, $K_{\mathrm{s}}-L$ could appear bluer than it is for close-by stars, moving the corresponding datapoints leftwards in the diagram, i.e., increasing the apparent number of sources without a NIR excess. 
F. Massi et al.: Testing circumstellar disk lifetimes in young embedded clusters associated with the Vela Molecular Ridge

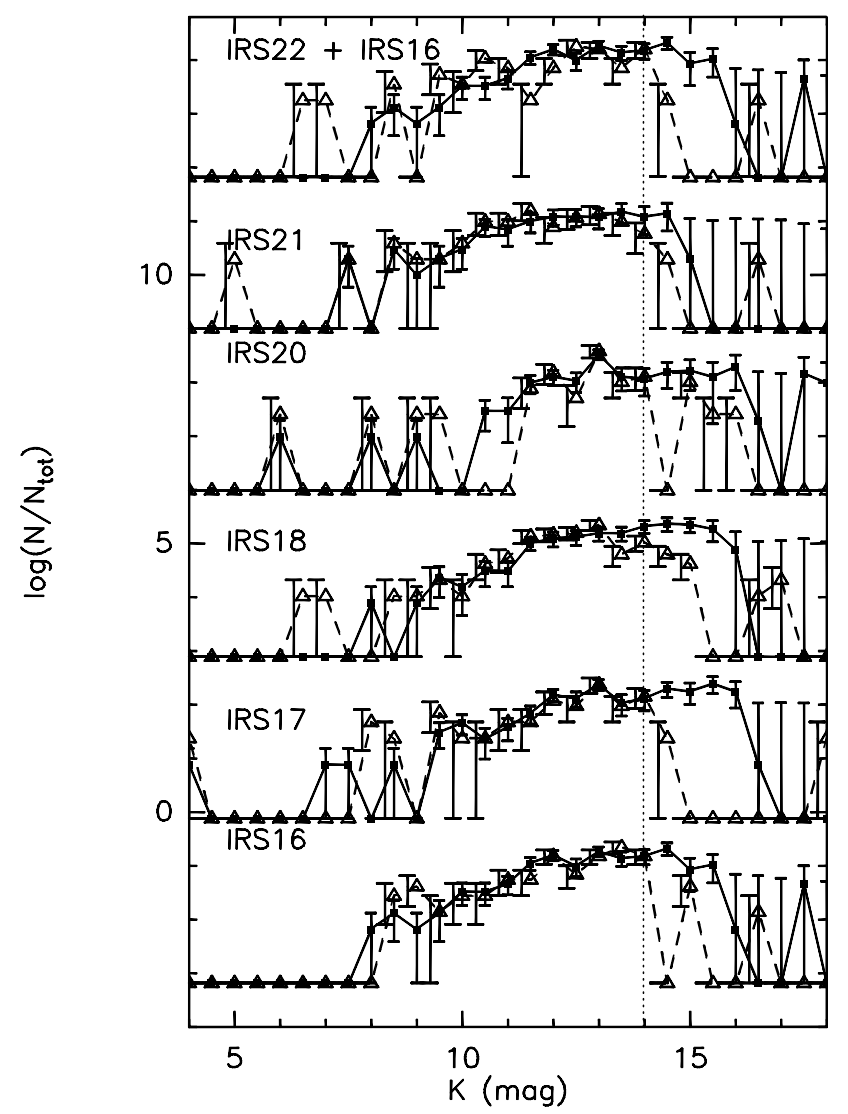

Fig. 10. Dereddened $K$ luminosity functions for the 6 clusters obtained by using all $K_{\mathrm{S}}$ sources with an $L$ counterpart (open triangles, dashed lines), overlaid with the corresponding dereddened $K_{\mathrm{s}}$ luminosity functions by Massi et al. (2006; full squares, solid lines). Errorbars for Poissonian errors are also drawn (those for the $L$ sources are slightly shifted along the $x$-axis). The vertical dotted line marks the dereddened completeness limit $\left(K_{\mathrm{s}} \sim 14\right)$ estimated by Massi et al. (2006) for their $K$ luminosity functions.

Furthermore, all sources falling above the reddening band of the main sequence have been conservatively counted as stars without a NIR excess.

As discussed in Sect. 3.5, the contamination by field stars is expected to be quite low. It can be estimated by noting that our photometry for stars with $L<14.25$ appears to sample all sources to a dereddened $K_{\mathrm{s}}=14$. By counting all objects to $K_{\mathrm{s}}=14$ in the dereddened KLFs for field stars estimated by Massi et al. (2006) and scaling their number to the area of the $L$ fields, we derived 5-6 contaminating stars per field. This agrees with what is found in Sect. 3.5.

The fractions of stars with a NIR excess per cluster are listed in Table 2, both with and without correction for field stars. The quoted uncertainties are obtained by propagating the Poissonian errors. We derived the fractions both including all stars to $L_{\text {compl }}$ and including only stars to $L_{\text {compl }}^{\prime \prime}$, to test the effect of both the different completeness limits and the possible contamination of the NIR excess region from very low mass stars. We clearly obtain slightly higher fractions for the lower completeness magnitude. If these were to also be corrected for field star contamination by using the same numbers as above, they would become even higher. But the highest values are found for the clusters with fewer sources below $L_{\text {compl }}^{\prime \prime}$ (IRS17 and IRS20) and the contamination from field stars is now also overestimated. Hence, the differences for each cluster remain within the errors.

\subsection{Sources of bias in counting stars with a circumstellar disk}

There is a major effect that has to be taken into account when deriving the fraction of stars with a NIR excess using cuts in magnitude. Because of extinction, a cut in magnitude implies that the volume in which less massive stars are detected decreases with decreasing mass. This problem would be even worse if the age spread were not negligible. If stars of the same mass with and without a disk were detected within the same volume, even if this changed with mass there would be no problems in deriving a meaningful ratio. But, because of the NIR excess, stars with a circumstellar disk can be detected deeper in the cloud than stars of the same mass without a circumstellar disk. The number of stars with a NIR excess is therefore bound to overestimate the number of stars with a circumstellar disk used in computing the ratio. One can impose a raw limit in mass by selecting only sources with a dereddened $K_{\mathrm{s}}$ magnitude (see Sect. 3.5), $K_{\text {dered }}$, brighter than a limiting value. We computed new fractions by selecting only sources with $L<L_{\text {compl }}$ and $K_{\text {dered }}<14$; they are listed in Table 2, both with and without correction for field star contamination. The derived values appear to be slightly less than the others, although within the errors. Because the NIR excess is lower in $K_{\mathrm{s}}$ than in $L$, if it is small on average, then the degree of contamination is significantly reduced.

We can take advantage of our having observed similar clusters belonging to the same region and with the same instrumental setups, and safely assume that the fraction $\epsilon$ of missed stars without a disk is the same for all clusters. If $n_{2}$ is the measured number of stars without a NIR excess, the true number of stars without a NIR excess will be $n_{2} /(1-\epsilon)$. It is easy to see that the true fraction $f$ is given by $f_{\mathrm{m}} \times(1-\epsilon) /\left(1-f_{\mathrm{m}} \epsilon\right)$, where $f_{\mathrm{m}}$ is the measured fraction. Therefore, $f_{\mathrm{m}}$ being roughly the same, all derived fractions have to be corrected by the same factor, i.e., their comparison is meaningful. And even for a pessimistic $\epsilon=0.3$, $f_{\mathrm{m}}$ (given in Table 2 ) should be decreased by only $0.85-0.9$ to obtain $f$.

Since the $J H K_{\mathrm{s}}$ and $L$ observations have been carried out relatively far apart in time, source variability may also affect the derived fractions. This is discussed by Lada et al. (2000), who conclude that the fraction of stars falling in the excess region only because of the variability is at most negligible in the Trapezium cluster. Again, we expect that it affects all 6 clusters in an identical way.

\subsection{Fraction of stars with a circumstellar disk in the clusters}

Remarkably, 5 out of 6 clusters have the same fraction of members with a NIR excess, within the errors. Although still within the error, IRS21 exhibits a lower fraction. According to Massi et al. (2006), the $K_{\mathrm{s}}$ photometry of IRS21 has a $K_{\mathrm{s}}$ completeness magnitude $1 \mathrm{mag}$ brighter than that of the other clusters. The colour-colour diagram of IRS21 shows 8 datapoints with $K_{\mathrm{s}}-L>2$ and large photometric errors in $H-K_{\mathrm{s}}$, lying above the adopted boundary between stars without and stars with a NIR excess. Objects with such a large value of $K_{\mathrm{s}}-L$ (i.e., $>2$ ) are usually embedded protostars (see, e.g., Haisch et al. 2000; Lada et al. 2000). All of these 8 sources are quite faint in $H$ (with photometric errors $>0.2 \mathrm{mag}$ ) and undetected in $J$, so it is likely that their $H-K_{\mathrm{s}}$ has been overestimated. Dropping these sources from the counting increases the fraction of members with a NIR excess of up to 0.59 (down to $L_{\text {compl }}$ and after correction for field stars), whereas counting them as stars with a NIR excess increases the fraction to 0.66 . In summary, there is evidence that 
IRS21, as well, has the same fraction of stars with circumstellar disks as the remaining 5 clusters.

We also derived the fraction of stars with a NIR excess by imposing a lower $K_{\text {dered }}$ limit (i.e., $K_{\text {dered }}<11$ ) to select the highest mass stars in a cluster. The results are listed in Table 2. Although the statistics are low and contamination by foreground stars may not be negligible, the fraction of stars with a NIR excess is clearly lower. This would agree with disk lifetimes being shorter for higher mass stars. However, spectroscopic observations should be used to infer cluster membership for the brightest stars.

\subsection{Cluster age estimates}

In conclusion, our $J H K_{\mathrm{s}} L$ photometry of 6 young embedded clusters in Vela-D shows that the clusters host the same fraction of stars with a circumstellar disk. This reinforces the notion that all stars (at least the low-mass ones) have a circumstellar disk at birth that dissipates with time (by accretion onto the central star, dynamical interactions, photodissociation and/or evolving to a planetary system; for a review of the different mechanisms see Hollenbach et al. 2000). Interestingly, IRS16, the only cluster of the sample associated with an HII region, is not different in this respect. The ionisation source is an early B star (Massi et al. 2003) and this suggests that at least non-extreme UV environments (i.e., those that do not originate in O stars) do not significantly accelerate the dissipation of disks. This agrees with the estimated timescales for disk dissipation by UV radiation from external sources. The latter is $<10^{7}$ yrs only for the outer region of disks ( $>10 \mathrm{AU}$ ), which does not contribute much to the NIR excess, and in Trapezium-like clusters, as shown by Hollenbach et al. (2000).

Using the relation found by Haisch et al. (2001), a fraction of $0.6-0.7$, as observed in the 6 clusters, translates into an age of $\sim 2 \mathrm{Myr}$, in agreement with what found by Massi et al. (2006). Hillenbrand (2005) also derives the fraction of stars with a circumstellar disk in clusters from NIR colours, but in a slightly different way. By comparison with her results, we again obtain an age for the 6 clusters of 1-2 Myr. Interestingly, the presence of an HII region (263.619-0.533) associated with IRS16 can be used to constrain the age of the clusters. According to Caswell \& Haynes (1987), the size of the radio emission at $5 \mathrm{GHz}$ is $1^{\prime}$. This agrees with the size measured in the $K_{\mathrm{s}}$ image, which is $\sim 2^{\prime}$. Assuming a radius of $1^{\prime}$, this corresponds to $0.2 \mathrm{pc}$ at a distance of 700 pc. Massi et al. (2003) found that the ionising source is probably a B0-B2 V star. The Strömgren radius of an HII region produced by a B0-B1 ZAMS star is $r_{\mathrm{c}}=5.17-0.75 \times n_{3}^{-2 / 3} \mathrm{pc}$, where $n_{3}$ is the electron density in units of $10^{3} \mathrm{~cm}^{-3}$ (Churchwell \& Walmsley 1973). Hence, 263.619-0.533 does not appear to have reached the Strömgren radius, unless it expanded in a dense $\left(10^{4}-10^{5} \mathrm{~cm}^{-3}\right)$ gas that has now cleared away. Even in the case of very dense gas $\left(10^{5} \mathrm{~cm}^{-3}\right)$, the HII region would be larger than the Strömgren sphere only for an ionising star later than B1 V. Before reaching the Strömgren radius, an HII region expands at a speed exceeding the sound speed in an ionised medium, i.e., $>10 \mathrm{~km} \mathrm{~s}^{-1}$ (see e.g., Dyson \& Williams 1997). Hence, 263.6190.533 would have expanded to its current radius in $<2 \times 10^{6} \mathrm{yrs}$. Even in the case of a B1 star and a dense $\left(10^{5} \mathrm{~cm}^{-3}\right)$ medium, the dynamical time of the HII region would be short (in comparison to the time needed to reach the Strömgren radius), i.e., $4 \times 10^{4}$ yrs (Dyson \& Williams 1997). The dynamical age of the HII region is in all cases fully consistent with the inferred cluster age.
Given that the age of the clusters is constrained to be $\sim 10^{6} \mathrm{yr}$, it would be helpful and appears achievable to identify the physical process that can disperse the circumstellar disks around the sources on such a timescale. As for low mass stars, among the mechanisms discussed by Hollenbach et al. (2000) only viscous accretion onto the central stars seems to be efficient in dispersing the inner disks so rapidly. Stellar encounters are irrelevant for small clusters, the timescale being at least $\sim 10^{8}$ yrs according to Hollenbach et al. (2000) for the star densities measured by Massi et al. (2006). This is confirmed, e.g., by the simulations of Adams et al. (2006).

\section{Conclusions}

We have carried out imaging in the $L$ band $(3.78 \mu \mathrm{m})$ with ISAAC at the VLT, of 6 young embedded star clusters associated with cloud D of the Vela Molecular Ridge. These are in a similar evolutionary stage and, therefore, of the same age. The $L$ photometry has been complemented with $H K_{\mathrm{s}}$ photometry from SofI images. We used $H-K_{\mathrm{s}}$ versus $K_{\mathrm{s}}-L$ diagrams to derive the fraction of cluster members with a circumstellar disk. Our $L$ images have higher spatial resolution and no saturation problems compared to Spitzer/IRAC images. The main sources of bias and errors are carefully discussed. The main points of the present study are summarised as follows:

1. We revised the list of NIR infrared sources that had been associated with the IRAS point sources in each field.

2. By selecting $K_{\mathrm{s}}$ sources with $L$ counterparts, we constructed the cluster KLFs. Once dereddened by following the method outlined by Massi et al. (2006), we showed that these are statistically consistent with those obtained by Massi et al. (2006). This demonstrates that the $L$ images provide significant samples of the cluster populations and allowed us to estimate the degree of contamination from field stars.

3. Using different selection criteria (also based on the dereddened KLFs), we estimated a fraction of stars with a circumstellar disks $\sim 0.6-0.7$ that appears to be the same for all clusters, within the errors. This shows that the clusters host the same fraction of stars with a circumstellar disk, i.e., this fraction depends only on cluster age.

4. We showed that the impact of the main sources of bias is reduced significantly when observing clusters belonging to the same molecular cloud with the same instruments and instrumental setups, as in the present case.

5. The derived fraction of stars with a circumstellar disk constrains the age of the 6 clusters to be 1-2 Myr, refining previous age estimates.

6. The fraction of stars with a circumstellar disk appears to be lower for the most massive stars. This would agree with the shorter disk lifetimes of massive stars, although the results could be biased by the poor statistics of the available data.

Acknowledgements. L.V. is supported by the Basal Center for Astrophysics and Associated Technologies PFB-06 and by the Fondecyt project No. 1095187.

\section{Appendix A: Individual sources}

The observed fields were originally selected by Liseau et al. (1992), as sites of IRAS point sources with red colours. Massi et al. $(1999,2003)$ found that the fields are associated with young embedded clusters and that all IRAS uncertainty ellipses lie towards the centres of each cluster. These authors suggest that most of the FIR luminosity from each IRAS point source is contributed by protostars whose NIR counterparts lie within (or very 


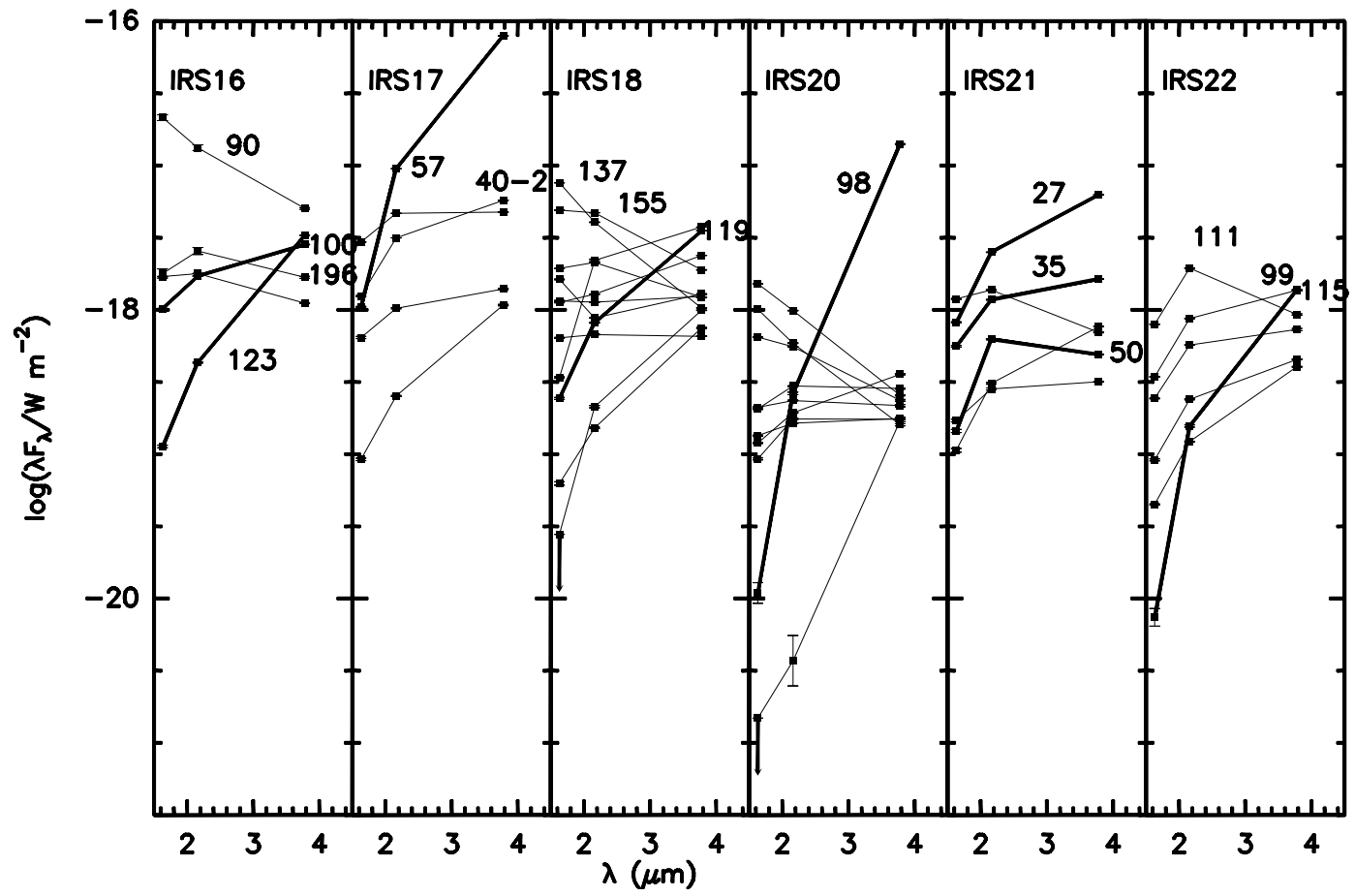

Fig. A.1. Spectral energy distributions for the brightest $L$ sources found in all 6 fields. The field name is at the top of each box. The steeply rising SEDs are indicated by thick lines. The most remarkable sources are labelled according to the catalogue numbers by Massi et al. (1999, 2003).

close to) the uncertainty ellipse and become brighter and brighter with increasing wavelengths. Hence, they should be quite distinctive in the $L$ band data. To check this, the NIR SEDs of the brightest $L$ sources in each imaged field are plotted in Fig. A.1. Clearly, all plots (one per field) exhibit at least one source with a steep SED whose flux increases with increasing wavelengths and dominates over the other objects in $L$. The $H K_{\mathrm{s}} L$ magnitudes were converted into fluxes following Mégessier (1995). A brief description of the 6 fields follows. Each field will be identified by using the IRS notation of Liseau et al. (1992).

IRS16 is different from the other 5 IRAS sources in the present sample, in that it was not considered as a "bona fide" Class I source by Liseau et al. (1992). It is indeed the only one associated with an HII region, i.e., 263.619-0.533 (Caswell \& Haynes 1987). Massi et al. (2003) show that the IRAS uncertainty ellipse is roughly centred on a B0-B2 star (their \# 90, see also Fig. 1). Massi et al. (2007) found that the IRAS uncertainty ellipse lies towards a minimum in 1.2-mm continuum emission, in the middle of three prominent peaks (their MMS1, MMS2, and MMS3). It is possible that the FIR emission originated in an UCHII region close to the central B star. The source exhibiting the steepest rising SED in Fig. A.1, is \# 123 of Massi et al. (2003), but it lies outside the uncertainty ellipse. Another NIR source with a rising SED is \# 100 of Massi et al. (2003), which lies within the IRAS ellipse (see Fig. 1). Both were already identified in $J H K$ by Massi et al. (2003).

The NIR counterpart of IRS17 is clearly the one already proposed by Massi et al. (1999), i.e., their \# 57. Giannini et al. (2005) showed that this NIR source lies towards a dense molecular core found in 1.2-mm continuum emission (MMS4 of Massi et al. 2007). They also searched for the driving source of a prominent jet, which lies towards a group of NIR sources outside the IRAS uncertainty ellipse, on the west. This group coincides with source \# 40 of Massi et al. (1999, see also Fig. 2), which was resolved into more than one star in ISAAC NIR images presented by Giannini et al. (2005). Of these, \# 40-2 is bright in $L$ and exhibits a rising SEDs (Fig. A.1).

As for IRS18, our $L$ image again confirms that the NIR counterpart to the IRAS source is the one already identified by Massi et al. (1999), i.e., their \# 119. This exhibits a rising SED and is one of the brightest $L$ sources in the field (see Fig. A.1).

There is no doubt that the NIR counterpart of IRS20 is the one already identified by Massi et al. (1999), i.e., their \# 98. It displays a steeply rising SED and is by far the brightest $L$ source in the field (see Fig. A.1). Figure 4 indicates that \# 98 lies at the centre of a bipolar nebula. Giannini et al. (2007) found a bipolar jet in the $\mathrm{H}_{2} 2.12-\mu \mathrm{m}$ emission, centred on \# 98 and aligned with the nebula, suggesting that it is driven by \# 98. This is also consistent with this source being viewed through a disk edge-on, as suggested by Massi et al. (2003).

In contrast, Fig. A. 1 excludes \# 50 of Massi et al. (1999) being as proposed by these authors the NIR counterpart of IRS21, which is within the IRAS uncertainty ellipse. The most suited candidate appears to be \# 27 of Massi et al. (1999), which however is located outside the IRAS ellipse (see Fig. 5). Source \# 35 of Massi et al. (1999) also exhibits a rising SED, but also lies outside the IRAS ellipse.

Figure A.1 suggests that the NIR counterpart of IRS22 might not be \# 111 of Massi et al. (2003), but that its photometry is affected by \# 111 consisting of two stars, unresolved in $H$ and $K_{\mathrm{s}}$. The most likely NIR counterpart is instead \# 115 of Massi et al. (2003), which exhibits a steeply rising SED and falls within the IRAS uncertainty ellipse (see Fig. 6). However, we cannot rule out that one of the two constituent sources of \# 111 has a steeply rising SED.

\section{References}

Adams, F. C., Proszkow, E. M., Fatuzzo, M., \& Myers, P. C. 2006, ApJ, 641, 504 Allen, C. W. 1976, Astrophysical Quantities, 3rd edn. (London: Athlone Press) 
Apai, D., Linz, H., Henning, Th., \& Stecklum, B. 2005, A\&A, 434, 987 Baba, D., Nagata, T., Nagayama, T., et al. 2004, ApJ, 614, 818 Baba, D., Sato, S., Nagashima, C., et al. 2006, AJ, 132, 1692

Baraffe, I., \& Chabrier, G. 1996, ApJ, 461, L51

Baraffe, I., Chabrier, G., Allard, F., \& Hauschildt, P. H. 1998, A\&A, 337, 403 Bessell, M. S., \& Brett, J. M. 1988, PASP, 100, 1134

Burkert, A., Stecklum, B., Henning, Th., \& Fischer, O. 2000, A\&A, 353, 153

Caratti o Garatti, A., Giannini, T., Lorenzetti, D., et al. 2004, A\&A, 422, 141

Carpenter, J. M. 2001, AJ, 121, 2851

Caswell, J. L., \& Haynes, R. F. 1987, A\&A, 171, 261

Churchwell, E., \& Walmsley, C. M. 1973, A\&A, 23, 117

D’Antona, F., \& Mazzitelli, I. 1994, ApJS, 90, 467

De Luca, M., Giannini, T., Lorenzetti, D., et al. 2007, A\&A, 474, 863

Dyson, J. E., \& Williams, D. A. 1997, The Physics of the Interstellar Medium, ed. R. J. Taylor, \& M. Elvis, 2nd edn. (Bristol \& Philadelphia: Institute of Physics Publishing)

Elia, D., Massi, F., Strafella, F., et al. 2007, ApJ, 655, 316

Giannini, T., Massi, F., Podio, L., et al. 2005, A\&A, 433, 941

Giannini, T., Lorenzetti, D., De Luca, M., et al. 2007, ApJ, 671, 470

Haisch, K. E. Jr., Lada, E. A., \& Lada, C. J. 2000, AJ, 120, 1396

Haisch, K. E. Jr., Lada, E. A., \& Lada, C. J. 2001, ApJ, 553, L153

Hillenbrand, L. A. 2005 [arXiv:astro-ph/0511083]

Hollenbach, D. J., Yorke, H. W., \& Johnstone, D. 2000, Disk dispersal around young stars, in Protostars and Planets IV, ed. V. Mannings, A. P. Boss, \& S. S. Russell (Tucson: The University of Arizona Press), 401

Kenyon, S. J., \& Hartmann, L. 1995, ApJS, 101, 117

Koornneef, J. 1983, A\&A, 128, 84

Lada, C. J., Muench, A. A., Haisch, K. E. Jr., et al. 2000, AJ, 120, 3162

Lada, C. J., \& Lada, E. A. 2003, ARA\&A, 41, 57
Liseau, R., Lorenzetti, D., Nisini, B., Spinoglio, L., \& Moneti, A. 1992, A\&A, 265,577

Lorenzetti, D., Spinoglio, L., \& Liseau, R. 1993, A\&A, 275, 489

Lorenzetti, D., Giannini, T., Vitali, F., Massi, F., \& Nisini, B. 2002, ApJ, 564, 839

Massi, F., Giannini, T., Lorenzetti, D., et al. 1999, A\&AS, 136, 471

Massi, F., Lorenzetti, D., Giannini, T., \& Vitali F. 2000, A\&A, 353, 598

Massi, F., Lorenzetti, D., \& Giannini, T. 2003, A\&A, 399, 147

Massi, F., Testi, L., \& Vanzi, L. 2006, A\&A, 448, 1007

Massi, F., De Luca, M., Elia, D, et al. 2007, A\&A, 466, 1013

Mégessier, C. 1995, A\&A, 296, 771

Moorwood, A., Cuby, J.-G., Ballester, P., et al. 1999, ESO Mess., 95, 1

Murphy, D. C., \& May, J. 1991, A\&A, 247, 202

Netterfield, C. B., Ade, P. A. R., Bock, J. J., et al. 2009, ApJ, 707, 1824

Olmi, L., Ade, P. A. R., Anglés-Alcázar, D., et al. 2009, ApJ, 707, 1836

Ortiz, R., Roman-Lopes, A., \& Abraham, Z. 2007, A\&A, 461, 949

Palla, F., \& Stahler, S. W. 1999, ApJ, 525, 772

Persson, S. E., Murphy, D. C., Krzeminski, W., Roth, M., \& Rieke, M. J. 1998, AJ, 116, 2475

Rieke, G. H., \& Lebofsky, M. J. 1985, ApJ, 288, 618

Scalo, J. 1998, in The IMF Revisited: a Case for Variations in the Stellar Initial Mass Function, Proc. of the 38th Herstmonceux Conf., ed. G. Gilmore, \& D. Howell, ASP Conf. Ser., 142, 201

Siess, L., Dufour, E., \& Forestini, M. 2000, A\&A, 358, 593

Thi, W.-F., van Dishoeck, E. F., Dartois, E., et al. 2006, A\&A, 449, 251

van der Bliek, N. S., Manfroid, J., \& Bouchet, P. 1996, A\&AS, 119, 547

Wouterloot, J. G. A., \& Brand, J. 1999, A\&AS, 140, 177

Yamaguchi, N., Mizuno, N., Saito, H., et al. 1999, PASJ, 51, 775 